\title{
VARIATION DIMINISHING TRANSFORMATIONS
}

BY DEBORAH TEPPER HAIMO

Communicated by R. P. Boas, October 28, 1963

The present note is a brief summary of the principal results of the author's doctoral dissertation at Harvard University on an inversion theory and a corresponding representation theory for convolutions of variation diminishing Hankel kernels. These results are parallel to and partly include the theory developed by Hirschman and Widder in [1]. Complete details with proofs will appear in a later publication.

Let $\gamma$ be a fixed positive number and let

$$
\mu(x)=\frac{x^{2 \gamma+1}}{2^{\gamma+1 / 2} \Gamma(\gamma+3 / 2)} .
$$

Denote by $L^{P}(0, \infty ; d \mu), 1 \leqq p<\infty$, the space of all real-valued measurable functions $f$ defined on $(0, \infty)$ for which the norm $\|f\|_{p}$ is finite where

$$
\|f\|_{p}=\left[\int_{0}^{\infty}|f(x)|{ }^{p} d \mu(x)\right]^{1 / p} .
$$

$L^{\infty}(0, \infty ; d \mu)$ denotes the space of those functions $f$ for which $\|f\|_{\infty}$ is finite where

$$
\|f\|_{\infty}=\underset{0<x<\infty}{\text { ess. l.u.b. }}|f(x)| \text {. }
$$

Define

$$
g(x)=2^{\gamma-1 / 2} \Gamma(\gamma+1 / 2) x^{1 / 2-\gamma} J_{\gamma-1 / 2}(x)
$$

and

$$
g(x)=2^{\gamma-1 / 2} \Gamma(\gamma+1 / 2) x^{1 / 2-\gamma} I_{\gamma-1 / 2}(x),
$$

where $J_{\gamma-1 / 2}(x)$ is the ordinary Bessel function and $I_{\gamma-1 / 2}(x)$, the Bessel function of imaginary argument. The Hankel transform $f^{\wedge}(x)$ of a function $f$ of $L^{1}$ is given by $f^{\wedge}(x)=\int_{0}^{\infty} g(x t) f(t) d \mu(t), 0 \leqq x<\infty$. Let

$$
G_{N}(x)=\int_{0}^{\infty} \frac{\mathcal{J}(x t)}{E_{N}(t)} d \mu(t), \quad 0 \leqq x<\infty, N=0,1,2, \cdots,
$$

where 


$$
E_{N}(t)=\prod_{k=N+1}^{\infty}\left(1+\frac{t^{2}}{a_{k}^{2}}\right), \quad N=0,1,2, \cdots,
$$

with $0<a_{1} \leqq a_{2} \leqq \cdots$ and $\sum_{k=1}^{\infty} 1 / a_{k}^{2}<\infty . G_{0}(x)$ is written simply as $G(x)$. Let $\Delta(x, y, z)$ be the area of a triangle with sides $x, y, z$ if such a triangle exists. Let

$$
D(x, y, z)=\frac{2^{3 \gamma-5 / 2}[\Gamma(\gamma+1 / 2)]^{2}}{\Gamma(\gamma) \pi^{1 / 2}}(x y z)^{-2 \gamma+1}[\Delta(x, y, z)]^{2 \gamma-2},
$$

if $\Delta(x, y, z)$ exists and zero otherwise. Define the associated function $f(x, y)$ of a function $f(x)$ of $L^{1}$ by

$$
f(x, y)=\int_{0}^{\infty} f(u) D(x, y, u) d \mu(u), \quad 0 \leqq x, y<\infty .
$$

It can be shown that the function $G_{N}(x, y)$ associated with $G_{N}(x)$ is given by

$$
G_{N}(x, y)=\int_{0}^{\infty} \frac{\mathcal{J}(x t) \mathfrak{J}(y t)}{E_{N}(t)} d \mu(t), \quad 0 \leqq x, y<\infty .
$$

For any two functions $f$ and $g$ of $L^{1}$, let

$$
f \# g(x)=\int_{0}^{\infty} f(x, y) g(y) d \mu(y), \quad 0 \leqq x<\infty .
$$

It follows that the space $L^{1}$, with multiplication defined by \#, forms a Banach algebra. For the particular convolution $G \# \phi(x)$ the following result holds:

Convergence THEOREM. Let $\phi$ be a function integrable in any finite interval, and let

$$
\int_{0}^{\infty} G\left(x_{0}, y\right) \phi(y) d \mu(y)=\lim _{T \rightarrow \infty} \int_{0}^{T} G\left(x_{0}, y\right) \phi(y) d \mu(y), \quad x_{0} \geqq 0,
$$

converge conditionally. Then

$$
\int_{0}^{\infty} G(x, y) \phi(y) d \mu(y)
$$

converges conditionally for all $x$ and uniformly for $x$ in any finite interval. In this respect $G \# \phi(x)$ behaves like the familiar Stieltjes transform.

If $H$ is a real-valued function of $L^{1}$ and $\phi$, a real-valued, continuous 
function of $L^{\infty}$, then $H$ is said to be a variation diminishing \#-kernel if and only if, for every such $\phi$, the number of variations of sign of $H \# \phi$ does not exceed the number of variations of sign of $\phi$. In [2], Hirschman generalized an earlier result of I. J. Schoenberg by proving that a \#-kernel $G$ is variation diminishing if and only if it has the form (1).

The main inversion theorem under the least restrictive hypotheses is the following:

INVERSION THEOREM. Let $\phi$ be a function integrable on every finite interval and let

$$
f(x)=\int_{0}^{\infty} G(x, t) \phi(t) d \mu(t), \quad 0<x<\infty,
$$

converge conditionally. Then

$$
\lim _{N \rightarrow \infty} \prod_{k=1}^{N}\left(1-\frac{\Delta_{x}}{a_{k}^{2}}\right) f(x)=\phi(x)
$$

where

$$
\Delta_{x} h(x)=h^{\prime \prime}(x)+\frac{2 \gamma}{x} h^{\prime}(x)
$$

if

$$
\lim _{h \rightarrow 0} \frac{1}{h} \int_{x}^{x+h}[\phi(t)-\phi(x)] d \mu(t)=0,
$$

a condition which holds almost everywhere.

The behavior of the variation diminishing kernels $G(x, y)$ and of the quotients $G_{N}(x, y) / G(x, y)$ of kernels plays a central role in the development of the theory. For example, the matrix $\left[G\left(x_{i}, y_{j}\right)\right]_{1 \leqq i, j \leqq n}$ is totally non-negative, where a real matrix is said to be totally nonnegative if and only if all its minors of any order are non-negative. From this it follows that $G(x, y) / G\left(x_{0}, y\right)$ is a monotonic decreasing function of $y$ for $x_{0}>x$ and monotonic increasing for $x_{0}<x$. The additional fact that

$$
\frac{G(x, y)}{G\left(x_{0}, y\right)} \sim \frac{\mathscr{I}\left(a_{1} x\right)}{\mathfrak{I}\left(a_{1} x_{0}\right)}, \quad y \rightarrow \infty,
$$

which follows from (2) by an appeal to the calculus of residues, leads to the proof of the convergence theorem stated earlier. 
The corresponding principal representation theorem is the following:

REPRESENTATION THEOREM. Necessary and sufficient conditions that a function $f$ be given by

$$
f(x)=\int_{0}^{\infty} G(x, t) d \psi(t),
$$

with $\psi(t) \uparrow$ are that

(i) $f(x) \in C^{\infty}, 0 \leqq x<\infty$

(ii) $f^{(2 k+1)}(0)=0, k=0,1,2, \cdots$,

(iii) $f(x)=o\left(g\left(a_{1} x\right)\right), x \rightarrow \infty$,

(iv) $\prod_{k=1}^{N_{i}}\left(1-\Delta_{x} / a_{k}^{2}\right) f(x) \geqq 0,0<x<\infty, 1=N_{0}<N_{1}<\cdots$.

The proof of this result depends on a fundamental representation theorem which states that a function $f$ satisfying conditions (i)-(iv) above is given by

$$
\begin{aligned}
f(x)=\int_{0}^{\infty} G_{N_{\imath}}^{*}(x, y)\left[\prod_{k=1}^{N_{i}}\left(1-\frac{\Delta_{y}}{a_{k}^{2}}\right) f(y)\right] d \mu(y), & \\
& 0<x<\infty, 1=N_{0}<N_{1}<\cdots,
\end{aligned}
$$

where

$$
G_{N}^{*}(x)=\int_{0}^{\infty} \frac{\partial(x t)}{\prod_{k=1}^{N}\left(1+\frac{t^{2}}{a_{k}^{2}}\right)} d \mu(t), \quad 0<x<\infty, N=1,2, \cdots
$$

This basic result in conjunction with an application of Helley's theorem and an appeal to Tauberian theorems serves to establish the main representation theorem.

\section{REFERENCES}

1. I. I. Hirschman, Jr. and D. V. Widder, The convolution transform, Princeton Univ. Press, Princeton, N. J., 1955.

2. I. I. Hirschman, Jr., Variation diminishing Hankel transforms, J. Analyse Math. 8 (1960-61), 307-336.

SOUTHERN ILLINOIS UNIVERSITY 